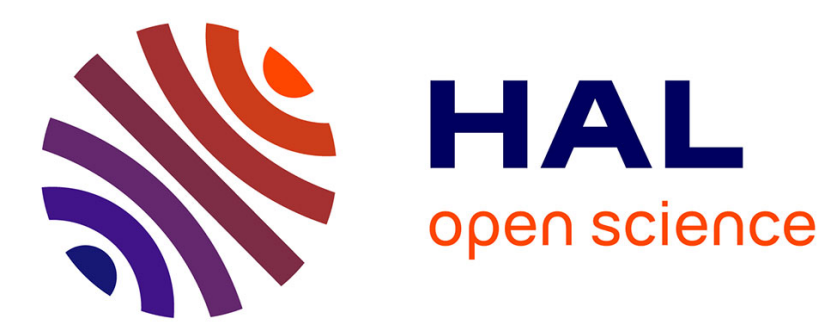

\title{
Entrez dans la danse avant d'y succomber
}

David Vasse

\section{To cite this version:}

David Vasse. Entrez dans la danse avant d'y succomber. Jean Grémillon et les quatre éléments, Presses universitaires du Septentrion, [9 p.], 2019, (Arts du spectacle. Images et sons), 978-2-75742446-9. hal-02157165

\section{HAL Id: hal-02157165 \\ https://hal-normandie-univ.archives-ouvertes.fr/hal-02157165}

Submitted on 15 Jun 2019

HAL is a multi-disciplinary open access archive for the deposit and dissemination of scientific research documents, whether they are published or not. The documents may come from teaching and research institutions in France or abroad, or from public or private research centers.
L'archive ouverte pluridisciplinaire HAL, est destinée au dépôt et à la diffusion de documents scientifiques de niveau recherche, publiés ou non, émanant des établissements d'enseignement et de recherche français ou étrangers, des laboratoires publics ou privés. 


\section{Entrez dans la danse avant d'y succomber}

David Vasse

Il est un fait qui n'échappe à aucun spectateur attentif du cinéma de Jean Grémillon : la présence, dans bon nombre de ses films de fiction, d'une scène de danse collective, ou plus exactement d'un état de crise individuel attesté par la manifestation dansée du collectif. Il y a en effet un moment où «faut que ça danse » chez Grémillon, pas seulement à dessein de libérer la convention d'une joie à partager indifféremment, par obéissance à un rituel populaire, mais surtout dans l'idée que la danse, dès qu'elle a lieu, excède les charmes de sa prodigalité festive pour atteindre au domaine de la fatalité.

Certes, faire danser des personnages à l'époque de Grémillon (en particulier à la veille des années 30 jusqu'au milieu des années 40) n’est pas chose rare dans le cinéma français. On pourrait même parler de figure imposée, diversement envisagée selon les périodes. Dans l'effervescence expérimentale des années 20, la danse apparaît comme le summum du mouvement déployé jusqu'à l'ivresse formelle, le plus inspirant en termes de plasticité et de montage. RicciottoCanudo, Abel Gance, Jean Epstein ou René Clair, tous ne cessent de faire implicitement ou explicitement référence à elle. «Classiquement, la danse donne un corps à l'affect, elle offre une apparence sensible à la pulsion, à l'affection, à l'émotion ${ }^{1}$. » C'est bien à ce titre qu'elle constitue une pointe de recherche rythmique majeure dans la production des avant-gardes. Dans le cinéma français des années 30, les occasions de danser ne manquent pas non plus. S'y multiplient les bals populaires, les guinguettes et les films-opérettes, tout un temps où l'on connait bien la chanson depuis l'impulsion enthousiaste des innovations sonores au tout début de la décennie. Sur les écrans français, la fête témoigne de deux phénomènes concomitants : d'un côté, la revalorisation du spectacle populaire, comme le cabaret ou le music-hall, grâce à l'exploitation des nouvelles techniques du son, de l'autre la volonté d'un renouveau social bientôt consacré par l'arrivée du Front Populaire en mai 1936 et les grèves de juin sur fond desquels culmine ce que Georges Sadoul nommait «1'école réaliste française ${ }^{2} \gg$.

\footnotetext{
${ }^{1}$ Nicole Brenez, De la figure en général et du corps en particulier (L'invention figurative au cinéma), Editions De Boeck Université, 1998, p. 301.

${ }^{2}$ Cité par Pierre Billard dans L'Âge classique du cinéma français (Du cinéma parlant à la Nouvelle Vague), Editions Flammarion, 1995, p. 220.
} 
Même s'il n'existe pas un « cinéma typique du Front populaire », force est de constater que plusieurs films français de cette période, y compris celle de la première moitié des années 30, conjuguent l'apport techniquement évocateur de la musique et de la chanson au progrès social, à la place accordée à la jeunesse et à l'humanisme fraternel. C'est ainsi que danser et chanter deviennent des actions récurrentes, presque une convention, le moment démocratique idéal dédié au peuple réuni avant la désillusion (les espoirs vite déçus du Front populaire, l'approche de la guerre). Il existe ainsi quelques films emblématiques au regard desquels la fête se présente comme le signe d'un partage des plaisirs de la vie simple ou au contraire de la vie cossue, ciment dans les deux cas d'une société qui trouve là matière à promouvoir ses valeurs et ses rites. Outre la trilogie de René Clair, décisive pour le parlant français, réalisée au tout début des années 30 (Sous les toits de Paris - 1930, Le Million - 1931 et A nous la liberté - 1931), il convient de citer Julien Duvivier, surtout La Belle équipe (1936) et Un carnet de bal (1937), Hôtel du Nord de Marcel Carné (1938) et son bal du 14 juillet, Dédé de René Guissard (1934) avec Danièle Darrieux et Albert Préjean, d'après une opérette créée en 1921 par Maurice Chevalier, et puis comment ne pas citer la fameuse fête macabre et prophétique de La Colinière dans La Règle du jeu de Jean Renoir (1939), film que le cinéaste avait défini d'une phrase si pertinente et si parlante au regard de cette époque sombre : «J'ai voulu filmer une société qui danse sur un volcan. »

Où et comment se situe Jean Grémillon par rapport à ces lois de l'attraction de la danse ? Comment l'envisage-t-il au sein de ses fictions ? Quel rôle dynamique et symbolique lui confie-t-il ? Quel type de présage contient chacune de ses interventions ? Une partie des réponses s'origine imaginairement dans la vocation de musicien de l'adolescent Grémillon, avec sa formation de violoniste à la Schola Cantorum de Paris puis son expérience de premier violon dans les orchestres d'accompagnement au Max Linder. On pourrait logiquement rattacher la présence ponctuelle de la danse à ce goût primordial et jamais démenti pour la musique, en tant qu'elle se situe au croisement du mouvement photogénique et de la temporalité affective. Seulement chez Grémillon, il importe de distinguer la danse de la chorégraphie par laquelle transitent généralement les notions d'unité et d'harmonie, la recherche méthodique d'un ordre plein et cohérent dont les vertus les plus spectaculaires se nomment la grâce, la fluidité, l'élan, au service d'un modèle parfait de séduction corporelle que la comédie musicale hollywoodienne a si superbement exalté. Pour Grémillon, il est moins question de mettre en scène la danse comme un absolu vers quoi tout converge, avec la promesse d'y voir la résolution miraculeuse de tous les conflits, que de filmer des 
personnages qui, à un moment donné, se mettent à danser pour affirmer, non pas la danse ellemême, mais ce qui en elle paradoxalement agit comme révélateur d'un défaut, d'une incomplétude ou d'une frustration. La danse n'est plus là pour effacer, dans l'amplitude de sa ronde endiablée, les tracas du quotidien, elle les stigmatise au contraire, au plus fort d'une tension entre l'abandon des corps et l'éveil du tourment. L'œuvre contient maints exemples dans lesquels la danse, art de l'équilibre, donne à ressentir une étrange et intenable combinaison entre une certaine revendication de liberté et de désir de fuir (au large ou dans le ciel) et les symptômes d'un poids qui l'emporte et détermine le sceau tragique d'une existence précipitée vers sa détresse. Au fond, la danse ne relie ni ne délie, elle marque une obstruction, un empêchement, une difficulté souvent fatale à concilier les êtres à leurs aspirations. C'est d'ailleurs ce qu'insinue régulièrement un élément plus ou moins volontaire de la part de Grémillon, à savoir un mobile d'interruption chargé de contrarier un peu plus l'événement, cette logique d'interruption qui du reste a marqué de manière lancinante la carrière de notre cinéaste.

\section{Maldone : la danse à l'état d'ivresse}

Dès Maldone, son premier long métrage de fiction (1927), on sait sur quel pied danse l'esprit sombre. Né de sa rencontre avec le comédien Charles Dullin qui lui propose d'adapter un roman d'Alexandre Arnoux, auteur souvent monté au Théâtre de l'Atelier, le film raconte l'histoire d'Olivier Maldone (Charles Dullin), vagabond qui a fui plusieurs années auparavant le château familial pour l'aventure des routes et les rencontres de hasard. Mais un drame - la mort accidentelle de son frère - va l'obliger, en tant qu'héritier, à regagner la propriété et à vivre une vie rangée et ennuyeuse. On trouve là une situation typique du cinéma de Grémillon, celle d'un personnage fidèle à un idéal qu'il juge sans compromis et qui à un moment est rattrapé par la pression d'un choix dont la cruauté est de mettre à l'épreuve ses plus profondes convictions. La force morale du personnage se mesure alors à l'aune de sa capacité de résistance, voire de rébellion, à ce qui s'oppose à son désir (s'exprime d'ailleurs dans cette attitude l'intégrité proverbiale du cinéaste: céder le moins possible aux compromis). Maldone avait quitté le château pour une autre vie, ses privilèges pour la liberté, et arrive le moment d'y renoncer par le rappel de cette réalité première.

En 1927, Grémillon est encore dans l'esprit de l'avant-garde. Il présente Tour au large au Vieux Colombier, réalise Photogénie mécanique, un montage conçu à partir de deux de ses 
anciens courts métrages documentaires, La Fabrication du fil et Du fil à l'aiguille. Puis, au cours d'une interruption du tournage de Maldone en octobre, il signe avec son opérateur Georges Périnal, Gratuités, un court métrage expérimental produit par Charles Dullin et composé de trucages optiques. Bref, lorsqu'il se lance dans l'adaptation du roman d'Alexandre Arnoux, il procède à la synthèse d'une narration à caractère naturaliste et de l'expérimentation visuelle. Il en ressort un film étonnant, déroutant, qui respecte les conventions du récit tout en les transfigurant par des options esthétiques proches de l'abstraction, où se croisent Jean Epstein et Abel Gance, en particulier dans les scènes à cheval - la balade en calèche qui dégénère et la course de Maldone à la fin, moments de folie qui font penser aux séquences les plus convulsives de La Roue (Abel Gance, 1922).

La scène dite de la Belle Marinière, du nom de la valse que Maldone interprète à l'accordéon lors du bal de la St-Jean du 24 juin 1927, propulse la danse dans un tourbillon de sensations extrêmes et paradoxales. C'est une scène caractéristique des opérations photogéniques effectuées par les cinéastes théoriciens des avant-gardes, fondées sur la vitesse, la scansion, la pure émotion plastique, avec un montage utilisé à dessein de faire fusionner deux fluides, le musical et le sentimental. Le roulier Maldone est amoureux de Zita la romanichelle (Génica Athanasiou), l'incarnation de son destin prédit par la voyante au début du film. Ce soir-là, elle danse sous ses yeux mais dans les bras d'un inconnu. Elle est emportée par la musique, si loin que Maldone s'emporte. Outre l'osmose qui existe entre le rythme endiablé de la musique et le rythme de la caméra et du montage, l'important est bien de hisser la danse à un niveau d'abstraction qui correspond au plus haut degré d'intensité du sentiment. Ce n'est plus la danse considérée de l'extérieur comme une activité commune inscrite dans le quotidien mais la danse comme ensemble de potentialités rythmiques directement connectées à une intériorité sous haute tension amoureuse. Durant cette période, Grémillon est très attaché à ce qu'il appelle «l'expression cinégraphique ». En 1925, il écrit : «C'est par ces abstractions mêmes, par ce mécanisme cérébral que notre création réduit la vie profonde et tumultueuse que nous cherchons, à des éléments d'intensité - aux puissances de nos impressions ${ }^{3}$. » Ici, l'enjeu est de pénétrer le tumulte de la passion par un dépassement du mouvement commun et de la perception.

Si la scène s'emballe jusqu'à la rupture, c'est en réalité pour deux raisons à connotation pessimiste reposant chacune sur le mauvais présage : l'entrée de Léonard (Georges Séroff),

3 Jean Grémillon, «Propositions » (1925), dans Le Cinéma? Plus qu'un art!, Editions L'Harmattan, 2010, p. 25. 
porteur de la mauvaise nouvelle et dépositaire d'une demande de retour au bercail, synonyme d'enfermement et de repli, et à l'opposé la liberté de Zita à prendre du plaisir dans des bras éphémères, ce qui rend Maldone non seulement fou de jalousie sur le moment mais aussi et déjà fou de ne pouvoir la rejoindre. Autrement dit, l'euphorie visuelle de la scène ne tient pas uniquement à l'intensité de l'instant amoureux ni à l'osmose décrite plus haut, mais bien au frottement infernal de deux temps incompatibles, le passé et l'avenir ; le passé qui rattrape Maldone et que Zita, dans l'absolu, refuse (un peu plus tard, dans la scène du bal du casino, symétrique à celle-ci, elle avouera à Maldone qu'elle peut fêter le passé mais pas vivre dedans) et l'avenir non tracé des routes et des charmes de l'existence que Zita veut préserver et dont Maldone se verra bientôt privé. Autrement dit, l'unité expérimentale de la danse ne consiste paradoxalement qu'en ce qui, en elle, agit comme des facteurs de séparation et d'éloignement, selon une pensée qui agite pareillement la grande séquence de la fête foraine dans Cour fidèle de Jean Epstein (1923).

\section{La Petite Lise : courber le destin au son de l'euphorie}

A la fin de La Petite Lise (1930), on assiste à une admirable conjonction de la temporalité physique introduite par la danse, plus exactement des corps dansants, et de la signature d'un destin figé à jamais. C'est le premier film parlant de Grémillon, le son y intervient régulièrement en contrepoint de l'image, comblant ce qu'elle ne peut montrer ou surgissant comme stridence au-delà de son seuil. C'est l'histoire poignante de Berthier (Alcover), un père «enfermé dehors » après une longue détention dans un pénitencier de Cayenne. On retrouve le schéma du personnage qui aspire à une vie différente (en l'occurrence une vie normale, avec travail et amour de sa fille retrouvée) mais qui finit par retourner à la case départ (en l'occurrence la case prison). On est de nouveau dans un scénario de la liberté compromise, littéralement (Berthier libéré de prison au début). C'est le cas aussi d'André (Bertheau) et de Lise (Nadia Sibirskaia) qui veulent partir vivre dans une petite maison mais qui n'ont pas d'argent pour réaliser ce rêve. Ils commettront le pire pour y arriver (le meurtre du receleur). Nous sommes à première vue dans un drame de type naturaliste avec la mise en place d'un déterminisme criminel et l'impossibilité d'échapper à son sort. Mais Grémillon réussit à transcender ces conventions par des choix d'une grande modernité, en particulier l'enregistrement de corps multiples (corps tatoués des bagnards au début, corps dansants du bal antillais à la fin) dont le flux d'énergie solidaire produit une durée qui fait masse, au 
mépris de l'efficacité narrative. C'est d'ailleurs l'un des préceptes du cinéma moderne, lorsqu'un poids de corps devient le principal instrument de mesure de l'expérience, opacifiant ainsi en s'y substituant toute psychologie (en ce sens, qu'un acteur massif comme Alcover joue le rôle de Berthier est fondamental). Si le film a été un grave échec public, c'est sans doute aussi pour ces raisons-là.

C'est la fin du film. Pour éviter la prison à sa petite Lise, Berthier endosse son crime et se dénonce à la police. Ce qui frappe dans cette fin, c'est bien sûr le détour prolongé par la danse, a priori en décalage par rapport à la gravité de la situation. Pourquoi Grémillon s'arrête-t-il aussi longtemps sur ces musiciens et ces danseuses alors que n'importe quel autre cinéaste serait resté sur le personnage, avec la fête en arrière-plan ? Ici, contrairement à Maldone, la fête se situe à côté du drame des personnages, hors-champ pourrait-on dire. Mais, plus profondément, elle boucle le film en répondant par le son et à distance aux bagnards torse nu du début, exactement comme Berthier retourne en prison. Là-bas on chantait, ici on danse, c'est le groupe qui, saisi dans sa communion, isole le personnage et insinue la fatalité de sa condition.

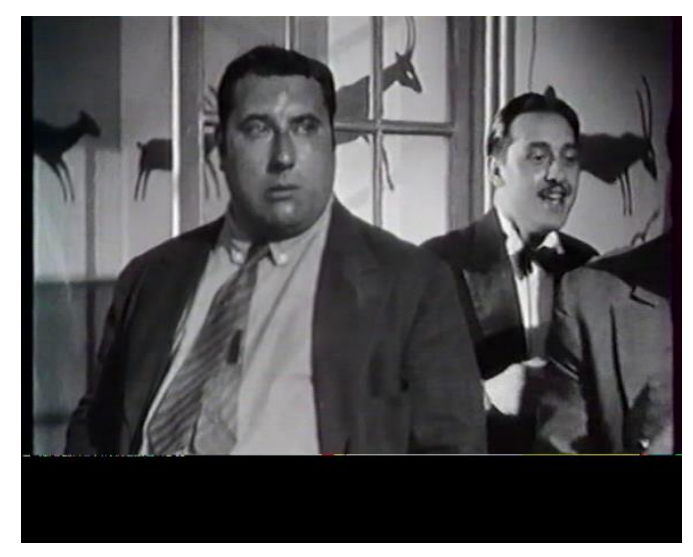

Tant par sa durée, d'essence documentaire, que par sa bande-son, cette fête fonctionne comme en écho à ce que Berthier avait quitté au début, c'est comme un appel du bagne en quelque sorte. Outre le contraste visible entre la soudaine apathie du père et le débordement joyeux des danseurs, écart cruel à la mesure de sa résignation, ce qu'il faut retenir dans ce passage dansé est donc bien sa valeur de présage, souligné par un étrange effet d'attraction rétroactive, mentalement suggéré un peu plus tôt par l'insertion du plan inaugural de l'entrée du pénitencier, avec les prisonniers filmés de dos. De nouveau, danser maintenant traduit l'imminence du regret à jamais. C'est faire tourner le cercle de l'inéluctable. Difficile alors de 
ne pas relever l'éloquence des deux partis pris qui concluent le plan : l'interruption sèche des chants et de la musique et le travelling arrière qui l'accompagne, si souvent utilisé par Grémillon dans d'autres films. En se retirant, la caméra accuse la distance à prendre désormais vis-à-vis d'un bonheur trop brièvement goûté.

\section{Remorques : tournez, tournez, petits nuages}

On a un bel exemple de ce recul technique et spécifique au terme de la grande séquence du repas de mariage qui ouvre Remorques (1939-41). Ce soir-là, on célèbre les noces d'un des membres de l'équipage du Cyclone, un remorqueur dirigé par le Capitaine Laurent (Jean Gabin), homme droit et intègre que l'amour bientôt fera vaciller. C'est lui, et personne d'autre, qui ouvre le bal, moment d'allégresse non dénué pourtant de mélancolie et d'inquiétude, ce qu'Yvonne (Madeleine Renaud), l'épouse d'André Laurent, nommera un «nuage » et que confirmeront l'interruption de la noce et plus largement la suite du film, notamment ces fameux nuages qui longent la plage aux deux extrémités de la grande séquence de la visite de la maison isolée par André et Catherine (Michèle Morgan).

Précisons que le roman de Roger Vercel dont est tiré le film s'ouvrait, lui, sur un enterrement. Jean Grémillon et Jacques Prévert, venu se joindre à Charles Spaak et André Cayatte pour la troisième version du scénario, lui ont préféré une scène de fête. Néanmoins, la tristesse n'est pas absente. Dans cette séquence, les nuages s'invitent à la fête, comme appelés par elle. Tantôt, ils la recouvrent, tantôt c'est elle qui reprend le dessus. A la fin de son discours, André rend sobrement hommage aux disparus du Cyclone puis change de ton avec le sourire («Assez causé, c'est la noce, on est venu pour rigoler. Il faut danser »). La danse est ici introduite comme un élément de joie fédérateur, capable de conjurer les petits malaises. Pourtant, à son rythme, surgissent des sensations d'éloignement qui atténuent soudain un bonheur partagé. Des effets de distance, au cœur de la valse, contrarient le principe d'union symbolisé par la noce. On observe par exemple à distance et en direct ce qui menace tout mariage, à savoir l'adultère dont le personnage de Tanguy (Charles Blavette) est ostensiblement victime. Puis c'est Yvonne, bien entendu, qui, malgré ses dénégations («Depuis dix ans, rien n'a changé, c'est comme au premier jour »), commence à se plaindre du temps qui passe et transforme en lointains souvenirs les moments heureux de son couple ( Il y a bien longtemps qu'on n'a pas dansé ensemble »). Dans l'esprit d'Yvonne, la danse résonne comme une évocation un peu 
amère de jours si paisibles et devenus trop rares. Elle danse, participe à la fête mais ses pensées la tiennent à l'écart.

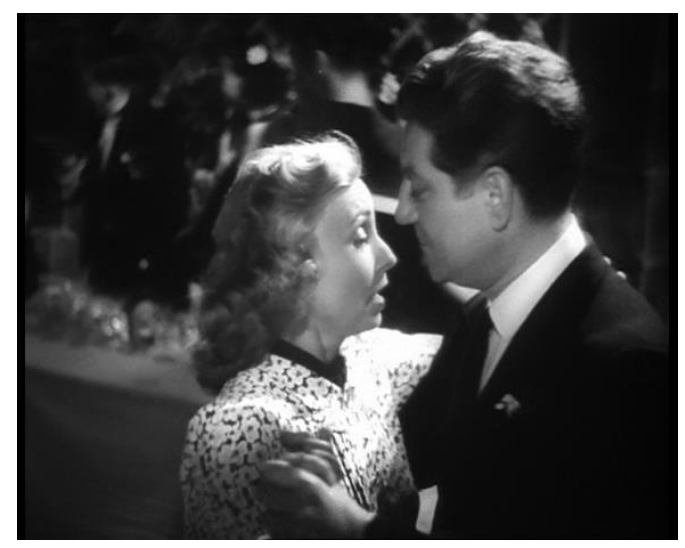

Comme dans Maldone, la joie collective est perturbée par la gravité intime qui s'y infiltre. On assiste de nouveau au contact des attractions : c'est dans la gaieté que s'éprouve la plus grande confusion mentale et sentimentale, jusqu'au pressentiment. Dès lors, tout est en place pour provoquer l'interruption de la noce par l'entremise du danger annoncé. L'équipage du Cyclone doit d'urgence reprendre du service pour remorquer un cargo perdu dans la tempête.

Chez Grémillon, beaucoup d'élans, d'espoirs, d'histoires d'amour, sont interrompus, souvent trop tôt, portant ainsi la marque de l'éphémère. Si Remorques est tragique, c'est aussi parce le film s'ouvre d'emblée sur une interruption et que cette interruption concerne également son tournage, démarré en juillet 1939 et achevé au début du mois de septembre 1941, sans cesse différé en raison d'événements eux aussi tragiques (le début de la guerre, l'incorporation de Grémillon comme sous-officier dans un régiment du Train, l'arrivée des troupes allemandes dans Paris le 14 juin 1940). J'aime à lire cette interruption de la noce et ce pressentiment d'Yvonne à l'aune de ce qui était en train de se tramer à l'époque : l'imminence du tragique et la fin du bonheur, une anticipation que figure parfaitement le dernier travelling arrière, juste avant l'arrivée à moto du porteur de SOS.

Tant de fois chez Grémillon, la danse est apparue comme l'attestation souvent vertigineuse d'un abandon, que celui-ci soit de l'ordre de l'ivresse, signe d'une émancipation audacieuse (Daïnah la métisse - 1931) ou au contraire d'une mortification sacrificielle, signe de renoncement ou de désaveu (voir la terrible scène finale de L'Etrange Madame X - 1951), la 
grande séquence de bal costumé de Lumière d'été (1942) réussissant, elle, à conjuguer ces deux mesures de l'abandon. On pourrait également citer le drame final de Pattes blanches (1948), en particulier la mort d'Odette (Suzy Delair), où le crime est monté en parallèle avec la fête nuptiale, comme si danser était une nouvelle fois une manière d'accompagner, au sens musical du terme, la précipitation inexorable du destin des personnages. C'est bel et bien dans ces moments fatidiques d'épanchement des corps que Jean Grémillon concilie idéalement sa passion pour la musique et les harmoniques de la passion. 